Lilianna Chęcińska*, Andrzej Jóźwiak, Magdalena Ciechańska, Carsten Paulmann, Julian J. Holstein, Birger Dittrich and Magdalena Małecka*

\title{
Quantifying intermolecular interactions for isoindole derivatives: substituent effect vs. crystal packing
}

https://doi.org/10.1515/zkri-2018-2076

Received February 20, 2018; accepted May 12, 2018; published online May 29, 2018

\begin{abstract}
The aim of the study was to examine noncovalent interactions in considerably different crystal packings of three isoindole compounds. Their structures were compared to three other closely-related derivatives described earlier in the literature. Here we discuss the crystal structures in the context of the hydrogen-bonded motifs and other weak interactions. The hierarchy of investigated intermolecular interactions was examined in a quantitative manner through pairwise interaction energies and energy framework analysis.
\end{abstract}

Keywords: crystal packing; energy frameworks; intermolecular interaction energy; isoindole derivatives; substituent effect.

\section{Introduction}

The term precision supramolecular engineering refers to the desired design of molecular solid state structures based on an understanding of intermolecular interactions. Supramolecular chemistry is of fundamental importance

\footnotetext{
*Corresponding authors: Lilianna Chęcińska and Magdalena Matecka, Department of Physical Chemistry, Theoretical and Structural Chemistry Group, Faculty of Chemistry, University of Lodz, Pomorska 163/165, 90-236 Lodz, Poland,

E-mail: lilianna.checinska@chemia.uni.lodz.pl (L. Chęcińska); malecka@uni.lodz.pl (M. Małecka)

Andrzej Jóźwiak and Magdalena Ciechańska: Department of Organic Chemistry, Faculty of Chemistry, University of Lodz, Tamka 12, 91-403 Lodz, Poland

Carsten Paulmann: Mineralogisch-Petrographisches Institut, Universität Hamburg, Grindelallee 48, Hamburg 20 146, Germany; and c/o HASYLAB/DESY, Notkestrasse 85, Hamburg 22 607, Germany

Julian J. Holstein: Department of Chemistry and Chemical Biology, TU Dortmund University, Otto-Hahn Str. 6, 44227 Dortmund, Germany

Birger Dittrich: Anorganische Chemie und Strukturchemie, Heinrich-Heine-Universität Düsseldorf, Düsseldorf, Germany
}

in both chemistry and biology due to the fact that structure determines function. Successful crystal engineering would depend on the control of directional noncovalent molecular interactions, which are exploited to form "supramolecular architectures" [1-3]. A wide variety of noncovalent interactions, such as hydrogen bonding and aromatic $\pi \cdots \pi$ stacking interactions, form the basis of an attempted design of supramolecular arrangements in organic crystals. Then there are the challenges of van der Waals type interactions where a strong preference for a preferred direction is missing, and the statistical nature of attractive interactions where several weak interactions can be competing. Therefore, it is important to study the hierarchy of interaction motifs and the topology of interaction energy.

The present study examines the properties of isoindole derivatives. These nitrogen-containing heterocycles occur in many natural products and biologically-active compounds [4-7]. This significant class of heterocyclic compounds attracts continuous attention due to a diverse pharmacological profile $[8,9]$. They can exhibit antimicrobial, anthelmintic and insecticidal properties; in addition, they have been attributed anticancer activity in reaction with the isoenzyme cyclooxygenase (COX-2), and thrombin inhibition. Moreover, further progress in heterocyclic chemistry depends on improving synthetic effects [4-6, 10-13].

The three isoindole compounds presented here were subjected to electron-density studies with a focus on weak intermolecular interactions in (aza)isoindole derivatives, especially those with a contribution of $\pi$-electrons [14]; earlier studies applied topological analysis using Bader's Atoms in Molecules theory (AIM) [15] and Hirshfeld surface analysis [16]. The present work uses a novel tool to compute model interaction energies [17] and to visualize their energy frameworks [18] with CrystalExplorer17 [19, 20]); this approach allows the intermolecular interaction energies to be calculated rather accurately, and provides a breakdown of the magnitude and significance of corresponding energy components analogous to the PIXEL approach [21]. 


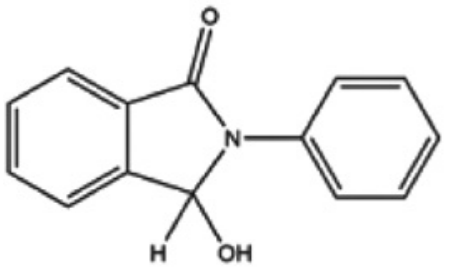

(I)

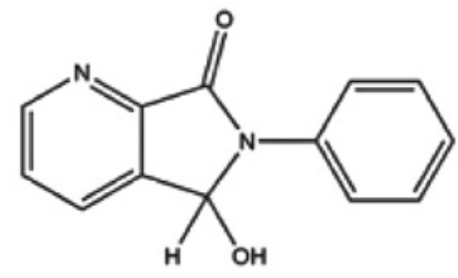

(II)

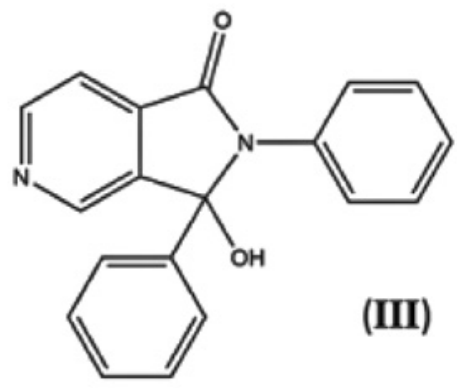

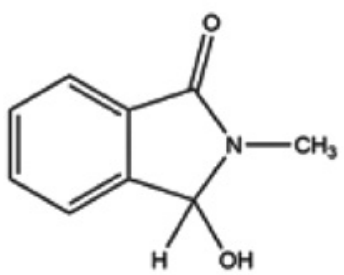

(IV)

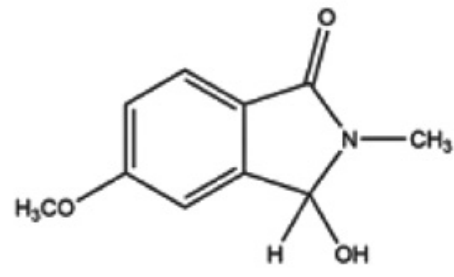

(V)

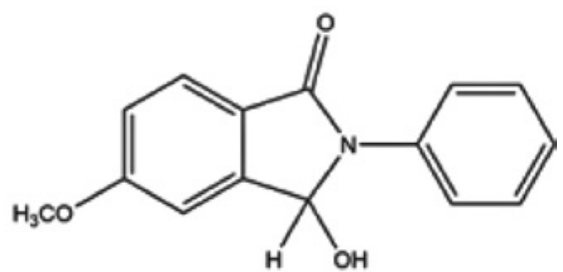

(VI)

Scheme 1: Molecular structures of the isoindole derivatives investigated previously (I)-(III) and in this study (IV)-(VI).

Our special interest is to relate isoindole compounds with different substituents to their crystal packing motifs. New isoindole derivatives differ by methyl, phenyl and methoxy group substituents, but are chemically closely related. Nevertheless they crystallize in considerably different packing arrangements. To understand the degree to which chemical substitution might affect crystal packing, various intermolecular interactions, "synthons" were identified in the crystal lattices, and quantified by energy decomposition used in CrystalExplorer17. To get a broader overview of the relevant structural features, the present study also includes results for three isoindole structures (I)-(III) studied previously (Scheme 1).

To enable a direct comparison of the new isoindole structures (IV)-(VI) with the high-resolution multipolar models of previously examined (aza)isoindole derivatives (I)-(III), the invariom approach $[22,23]$ was used; this approach allowed to improve significantly the structural models of (IV)-(VI) compared to independent atom model refinement. Modeling included elongating bond lengths to hydrogen atoms, so that they agree with neutron diffraction experiments [24], and provides a fragment-based electron-density distribution for medium or low-resolution X-ray datasets. The invariom formalism is a method based on the assumption that pseudoatoms are transferable from one molecule to another when the local chemical environment is the same. In the invariom approach, the multipole parameters are not refined, but are instead transferred from a database. These tabulated multipole parameters deposited in the database are obtained from density functional theory (DFT) calculations of defined model compounds with extended basis sets.

\section{Experimental}

\section{X-ray diffraction experiment}

The single crystal X-ray diffraction experiments for (IV)-(VI) were performed at beamline F1 of the storage ring DORIS III at the HASYLAB/DESY (Hamburg, Germany) on a kappa-geometry Huber diffractometer equipped with a MAR165 CCD detector. The temperature of the experiment was maintained at 100(2) K with an Oxford Cryosystem $\mathrm{N}_{2}$ gas-stream cooling device. The diffraction data were collected using synchrotron radiation at a choosen wavelength of 0.6000 (2) A. Cell refinement, data integration and reduction, and the scaling of diffracted intensities were carried out with the XDS software package $[25,26]$. In addition, the programs SADABS [27] or XPREP [28] were used merge the datasets to be used in the aspherical-refinement stages. Key crystallographic data regarding the studied compounds (IV)-(VI) are summarized in Table 1.

\section{Refinement and analysis}

All studied crystal structures of (IV)-(VI) were solved by direct methods using SHELXT [29] and initially refined using full-matrix least-square methods in SHELXL [30], both of which were accessed with the WinGX package [31]. All non-hydrogen atoms were refined anisotropically. All the hydrogen atoms were located in difference Fourier maps and refined freely with positional and isotropic displacement parameters. Following this, the InvariomTool software 
Tab. 1: Crystal data, data collection, spherical and invariom refinement of (IV)-(VI).

\begin{tabular}{|c|c|c|c|}
\hline & (IV) & (V) & (VI) \\
\hline \multicolumn{4}{|l|}{ Crystal data } \\
\hline Chemical formula & $\mathrm{C}_{9} \mathrm{H}_{9} \mathrm{NO}_{2}$ & $\mathrm{C}_{10} \mathrm{H}_{11} \mathrm{NO}_{3}$ & $\mathrm{C}_{15} \mathrm{H}_{13} \mathrm{NO}_{3}$ \\
\hline$M r$ & 163.17 & 193.20 & 255.26 \\
\hline Cell setting, space group & Monoclinic, $P 2_{1} / c$ & Orthorhombic, $\mathrm{Pbca}$ & Monoclinic, $P 2_{1} / c$ \\
\hline$a, b, c(\AA)$ & $7.967(2), 13.432(3), 8.352(2)$ & $14.046(3), 6.996(1), 18.442(4)$ & 13.374(3), 6.918(1), 13.245 \\
\hline$\beta\left(^{\circ}\right)$ & $118.24(3)$ & & $101.79(3)$ \\
\hline$V\left(\AA^{3}\right)$ & $787.4(3)$ & $1812.2(6)$ & $1199.6(4)$ \\
\hline Z & 4 & 8 & 4 \\
\hline$D_{\mathrm{x}}\left(\mathrm{Mg} \mathrm{m}^{-3}\right)$ & 1.376 & 1.418 & 1.413 \\
\hline Temperature (K) & 100 & 100 & 100 \\
\hline Radiation type $(\AA)$ & $0.6000(2)$ (synchrotron) & $0.6000(2)$ (synchrotron) & $0.6000(2)$ (synchrotron) \\
\hline$\mu\left(\mathrm{mm}^{-1}\right)$ & 0.098 & 0.105 & 0.099 \\
\hline Crystal form, color & Prism, colorless & Plate, colorless & plate, colorless \\
\hline Crystal size (mm) & $0.6 \times 0.4 \times 0.3$ & $0.6 \times 0.4 \times 0.2$ & $0.6 \times 0.5 \times 0.2$ \\
\hline \multicolumn{4}{|l|}{ Data collection } \\
\hline Diffractometer & $\begin{array}{l}\text { MAR165 CCD detector } \\
\text { at kappa-axis Huber } \\
\text { diffractometer }\end{array}$ & $\begin{array}{l}\text { MAR165 CCD detector } \\
\text { at kappa-axis Huber } \\
\text { diffractometer }\end{array}$ & $\begin{array}{l}\text { MAR165 CCD detector } \\
\text { at kappa-axis Huber } \\
\text { diffractometer }\end{array}$ \\
\hline Data collection method & $\varphi$ scans & $\varphi$ scans & $\varphi$ scans \\
\hline No. of measured and unique reflections & $64,984,3798$ & $63,104,2223$ & $58,006,5578$ \\
\hline Observed data with $F^{2} \geq 4 \sigma\left(F^{2}\right)$ & 3648 & 2018 & 5226 \\
\hline$R_{\text {int }}$ & 0.0298 & 0.0387 & 0.0276 \\
\hline$\theta_{\max }\left({ }^{\circ}\right)$ & 30.00 & 23.54 & 30.00 \\
\hline $\sin \theta_{\max } \backslash \lambda\left(\AA^{-1}\right), d(\AA)$ & $0.83,0.6$ & $0.66,0.75$ & $0.83,0.6$ \\
\hline Overall completeness to $\theta_{\max }(\%)$ & 99.5 & 99.0 & 96.1 \\
\hline Redundancy & 17 & 28 & 10 \\
\hline \multicolumn{4}{|l|}{ Spherical refinement (SHELX) } \\
\hline Refinement on & $F^{2}$ & $F^{2}$ & $F^{2}$ \\
\hline All data & 3798 & 2223 & 5578 \\
\hline$R, w R[I \geq 2 \sigma(I)]$ & $0.0363,0.1074$ & $0.0397,0.0965$ & $0.0326,0.0967$ \\
\hline$R, w R$ (all data) & $0.0370,0.1081$ & $0.0435,0.0986$ & $0.0344,0.0985$ \\
\hline Goodness of fit & 1.034 & 1.078 & 1.049 \\
\hline No. of parameters & 145 & 171 & 224 \\
\hline$\Delta \rho_{\max }, \Delta \rho_{\min }\left(\mathrm{e} \AA^{-3}\right)$ & $0.488,-0.304$ & $0.235,-0.297$ & $0.518,-0.240$ \\
\hline \multicolumn{4}{|c|}{ XD2006 Refinement after invariom assignment } \\
\hline Refinement on & $F[F>3 \sigma F]$ & $F[F>3 \sigma F]$ & $F[F>3 \sigma F]$ \\
\hline All data & 3729 & 2187 & 5496 \\
\hline Data included in refinement $\left(N_{\text {ref }}\right)$ & 3238 & 2072 & 5348 \\
\hline$R(F), R_{a l l}(F), R_{w}(F)$ & $0.0185,0.0204,0.0283$ & $0.0262,0.0286,0.0229$ & $0.0181,0.0187,0.0217$ \\
\hline$R\left(F^{2}\right), R_{\text {all }}\left(F^{2}\right), R_{w}\left(F^{2}\right)$ & $0.0284,0.0286,0.0562$ & $0.0415,0.0417,0.0458$ & $0.0262,0.0262,0.0426$ \\
\hline Goodness of fit & 4.56 & 3.61 & 3.31 \\
\hline No. of parameters & 118 & 138 & 185 \\
\hline$N_{\text {ref }} / N_{\mathrm{v}}$ & 27.44 & 15.01 & 28.91 \\
\hline$\Delta \rho_{\max }, \Delta \rho_{\min }\left(\mathrm{e} \AA^{-3}\right)$ & $0.175,-0.197$ & $0.204,-0.150$ & $0.104,-0.162$ \\
\hline
\end{tabular}

[32] was applied to obtain a non-spherical electron-density model by introducing invarioms. Using XD2006 [33], the positional parameters and atomic displacement parameters (ADPs) were again refined for non-hydrogen atoms. The $\mathrm{O}-\mathrm{H}$ and $\mathrm{C}-\mathrm{H}$ distances were elongated according to standard neutron distances and fixed. Only the isotropic $U_{\text {iso }}$ displacement parameters for each $\mathrm{H}$-atom were refined freely [34]. Refinement details are given in Table 1 . The residual density plots after invariom refinement in the plane of the isoindole moiety are displayed in Figure S1 of the Supplementary Material.
To identify molecular geometries and hydrogen-bond patterns, PLATON [35] and MERCURY [36] were used. Topological properties from the invariom models were obtained using the XDPROP subprogram in the XD package. Table S1 lists the electron density at the bond critical points and its Laplacian value for the experimental model compared with the theoretical models th-sp and th-opt (see Theoretical calculations, below).

Crystallographic data for (IV)-(VI) have been deposited with the Cambridge Crystallographic Data Centre as supplementary publications CCDC 1824398-1824400, respectively. Copies of the data can 
be obtained free of charge on application to CCDC, 12 Union Road, Cambridge CG21, EZ, UK [fax: (44) 1223-336-033; e-mail: deposit@ ccdc.cam.ac.uk].

\section{Theoretical calculations}

To verify the experimental model, single-point calculations were performed based on the geometries from the crystal structures of (IV)-(VI) (th-sp model). Additionally, full geometry-optimisations were also performed (th-opt model). All computations were carried out using Gaussian09 [37] at the B3LYP/6-311++G(3df,3pd) level of theory to make them directly comparable with previously-performed calculations on compounds (I)-(III). Subsequently, AIMAll [38] was used to evaluate the theoretical wave functions to obtain topological parameters according to the AIM theory.

\section{Model energy calculations}

Pairwise model energies were estimated between molecules within a cluster of a radius of $8 \AA$, using CrystalExplorer17 [19, 20] and Gaussian09 [37]. The computational approach uses an efficient procedure for obtaining the energy of intermolecular interactions in crystals. A suitably parameterized and simplified model (named CE-B3LYP) uses a B3LYP/6-31G $(\mathrm{d}, \mathrm{p})$ molecular wave function calculated based on the respective molecular arrangement in the crystal. The total interaction energy between any nearest-neighbor molecular pairs is estimated in terms of four components: electrostatic, polarization, dispersion and exchange-repulsion, with scale factors of 1.057, 0.740, 0.871, and 0.618 , respectively. In the present study, the crystal geometries of (IV)-(VI) were taken from the invariom procedure, bond lengths to hydrogen atoms were normalized to standard neutron values. For the individual derivative, the $8 \AA$ cluster gave 40,48 and 44 surrounding molecules for compounds (IV), (V) and (VI). Regarding the previously-reported compounds, clusters consist of 42, 47 and 40 molecules for compounds (I), (II) and (III). In the energy framework visualization procedure, pairwise energies are represented as cylinders joining the centers of mass of the molecules, where the cylinder thickness is proportional to the magnitude of the interaction energy.

As the intermolecular interactions in these crystal structures were expected to be dominated by dispersion forces, larger clusters than usual were created.

\section{Results and discussion}

\section{Molecular structures}

The molecular structures of the three isoindole derivatives (IV)-(VI) are displayed in Figure 1 together with the numbering scheme used. All molecules considered in our study (I)-(VI) consist of condensed 6- and 5-membered rings constituting an isoindole moiety, which is approximately planar (corresponding dihedral angles between mean planes are smaller than $3^{\circ}$ ), as well as a carbonyl oxygen (01) atom and hydroxyl group attached to the asymmetric carbon atom (C8); in addition, the variable substituents were present, which allow differentiation of the molecular structures, and the following functional groups are attached: a phenyl group to the $N$-isoindole atom (N1) in (I)-(III) and (VI), a methyl to the (N1) in (IV)$(\mathrm{V})$, a methoxy group to the C5-carbon atom in (V)-(VI), and a phenyl group to the asymmetric carbon atom (C8) and an azaisoindole-nitrogen atom (N2) in (II)-(III).

In the first part of the crystal structure analysis the molecular geometries of (IV)-(VI) were examined and compared with features of the previously described derivatives (I)-(III). As shown in Figure 2, the new molecular structures of (IV)-(VI) differ from each other with regard to the position of the hydrogen atom of the hydroxyl group; it is oriented downwards in (VI), but upwards in (IV)-(V). The latter orientation seems to be preferred, being observed in four molecules from six closely-related isoindoles; the hydroxyl group in structure (II) was found to demonstrate similar behavior to that in (VI). Another noticeable difference between the investigated compounds substituted by the $N$-phenyl group (Figure 2) is in the way the ring is bent with respect to the isoindole moiety, as reflected in the dihedral angle being $46.79(1)^{\circ}$, $20.01(1)^{\circ}, 55.98(1)^{\circ}$ and $17.13(1)^{\circ}$ for compounds (I), (II), (III) and (VI), respectively. In addition, the detailed analysis of bond lengths and corresponding electron densities at
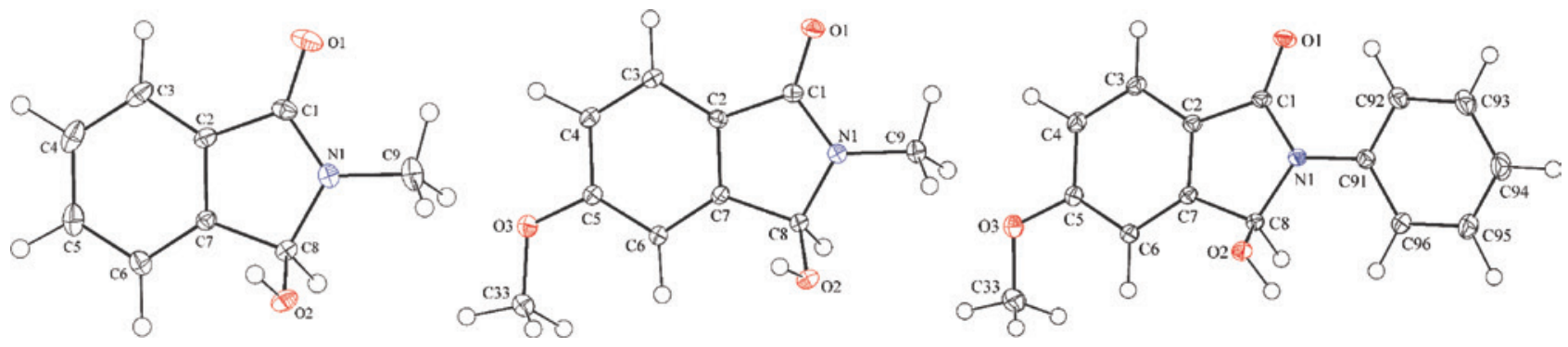

Fig. 1: Molecular structures of (IV)-(VI) with atom-numbering schemes. The anisotropic displacement parameters after invariom refinement are shown at the $50 \%$ probability level. Hydrogen atoms are drawn as spheres of arbitrary radius. 


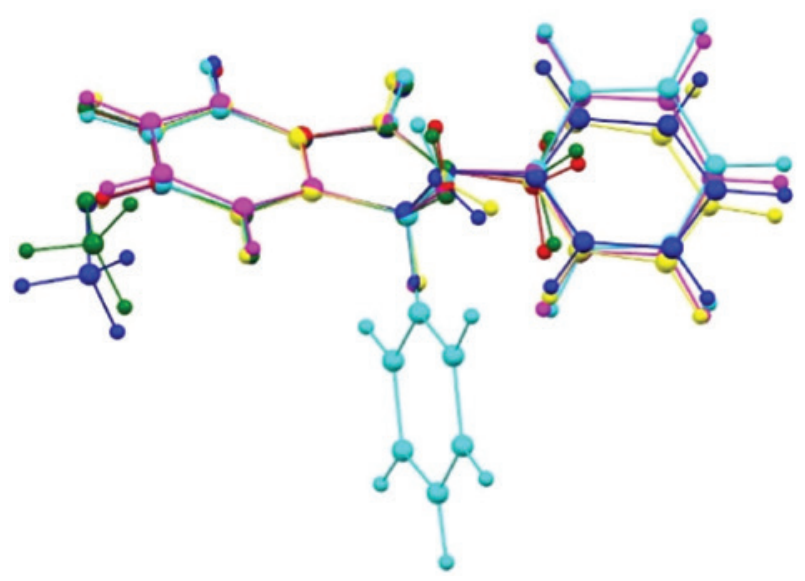

Fig. 2: Molecular overlays of isoindole derivatives: (I) - magenta, (II) - yellow, (III) - cyan, (IV) - red, (V) - green, (VI) - blue.

bond critical points (bcps) (Table S1 in the Supplementary Material) confirms that the N1-environment is affected by different substituents and/or crystal environments. The N1-C bond lengths were found to be in the range of

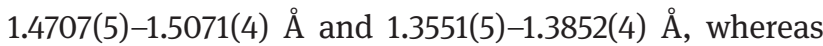
the corresponding electron densities at bcps range from 1.88 to 1.59 (2) e $\AA^{-3}$ for the N1-C8 bond, and from 2.26 to $2.08 \mathrm{e}^{-3}$ for the $\mathrm{N} 1-\mathrm{C} 1$ bond. These findings indicate that the electron-density values differ significantly from the average spread in the range of $0.1 \mathrm{e} \AA^{-3}$ indicated for transferability of bond topological properties from experimental charge-density determinations [39]. Since the results of the topological analysis is a result of the assigned invarioms for (IV)-(VI), the observed discrepancies cannot be discussed. However, the same trend of different bond length can be seen for gas-phase optimized structures; although, these differences are much more enhanced in the crystal structures. For example, the N1-C8 bond is the shortest in derivatives (IV) and (V) with a methyl group attached to an $\mathrm{N}$-isoindole atom, and the longest in structure (III), with two phenyl groups at the $\mathrm{N} 1$ and C8 atoms. Nevertheless, the five-membered ring remains flat and the overall skeleton of all the investigated molecules described herein have a similar conformation.

\section{Hirshfeld surface analysis}

The similarities and differences in the crystal structures of isoindole derivatives (I)-(VI) are visually summarized by Hirshfeld surface analysis. Hirshfeld surface fingerprint plots [40] for (I)-(VI) are depicted in Figure S2. Very sharp spikes can be clearly seen in all cases; these depict the formation of strong $\mathrm{O}-\mathrm{H} \cdots \mathrm{O}$ hydrogen bonds, as

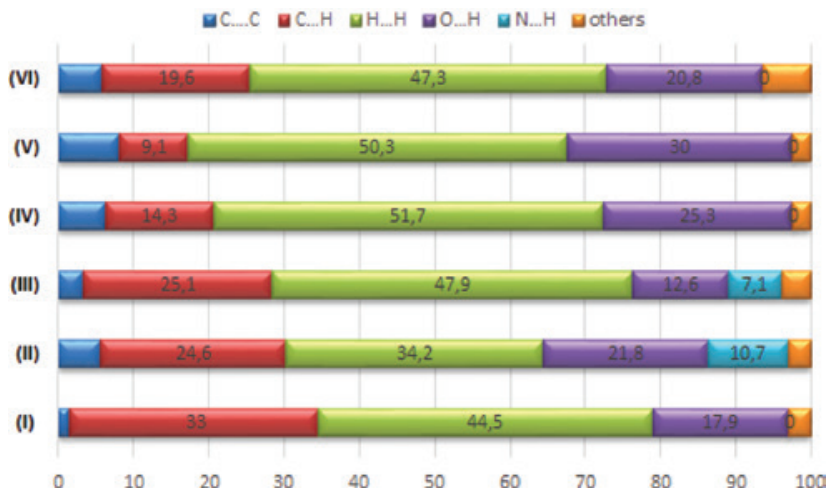

Fig. 3: Percentage contributions of various intermolecular contacts to the Hirshfeld surface area of compounds (I)-(VI).

well as $\mathrm{O}-\mathrm{H} \cdots \mathrm{N}$ in the case of (II). Hirshfeld fingerprint breakdown [41] (Figure 3) shows that $\mathrm{H} \cdots \mathrm{H}$ contacts dominate the Hirshfeld surface for all compounds, with a percentage contribution of around $50 \%$. The percentage contribution of $\mathrm{C} \cdots \mathrm{C}$ and $\mathrm{C} \cdots \mathrm{N}$ contacts (characteristic of aromatic stacking interactions) is similar with around $8 \%$; however, the contribution of these interactions is smaller for structures (I) and (III), and only $2 \%$ in the case of (I). Figure 3 indicates the presence of $\mathrm{N} \cdots \mathrm{H}$ contacts in azaisoindoles (II)-(III) due to the presence of an additional $\mathrm{N}$-atom of the isoindole moiety. In the presented pattern of interactions the percentage contribution of $\mathrm{H} \cdots \mathrm{H}$ is of equal magnitude to the sum of the percentage contributions of $\mathrm{C} \cdots \mathrm{H}$ and $\mathrm{O} \cdots \mathrm{H}$ contacts (around 40-50\%). Structure (III) differs most from all others, with a relatively small contribution of $\mathrm{H} \cdots \mathrm{H}$ interactions to the Hirshfeld surface, whereas structure (V) exhibits the greatest percentage discrepancy between the contributions of the $\mathrm{C} \cdots \mathrm{H}$ and $\mathrm{O} \cdots \mathrm{H}$ contacts. Despite the structural similarity percentage contributions are rather dissimilar and no clear conclusion emerges. Therefore a detailed analysis of the intermolecular packing features is presented next.

\section{Intermolecular interactions}

To understand their non-covalent interactions, the crystal packing of the studied derivatives was further examined. The intermolecular interactions and architecture of the crystal packing of (IV)-(VI) were first characterized from the geometrical point of view, and then by Hirshfeld surface analysis and energy framework calculations. Hirshfeld surface analysis has been found to be an invaluable tool in structural studies performed over the last decade $[42,43]$. The energy framework approach is a newer 
technique, but an increasing number of studies have indicated that it might also be a very powerful one [20, 44-46].

Compound (IV): The molecules of compound (IV) are linked by an intermolecular $\mathrm{O} 2-\mathrm{H} 2 \cdots \mathrm{O} 1(1-x,-y,-z)$ hydrogen bond, which is responsible for the formation of cyclic dimers about a center of symmetry (Figure 4). Such hydrogen-bonded dimers are additionally stabilized by aromatic $\pi \cdots \pi$ interactions between partially overlapping isoindole rings (Figure 4). Based on the geometrical parameters describing aromatic $\pi \cdots \pi$ interactions presented in Table S3, it can be seen that the electrons of five-membered rings within dimers interact with each other. The isoindole moieties of (IV) show a more efficient overlap between adjacent dimers. However, in this case, the five-membered rings are quite far away. Figure 4 presents the stacks of dimers propagating along the crystallographic $a$ direction (see blue ellipse/circle highlighting the stacks). Figure 5 presents the complete crystal packing in the unit cell.

In the crystal, weak $\mathrm{C}-\mathrm{H} \cdots \mathrm{O}$ interactions are

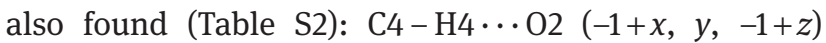
$\mathrm{C} 5-\mathrm{H} 5 \cdots \mathrm{O} 1(-x, 1 / 2+y,-1 / 2-z), \mathrm{C} 8-\mathrm{H} 8 \cdots \mathrm{O} 2(-x,-y$, $1-z)$. The first two interactions produce infinite chains of molecules running along the [101] and [010] directions, respectively (Figures S3-S4 in the Supplementary Material). In turn, the $\mathrm{C} 8-\mathrm{H} 8 \cdots \mathrm{O} 2(1-x,-y, 1-z)$ interaction is responsible for creating hydrogen-bonded dimers, which together with the $\mathrm{O}-\mathrm{H} \cdots \mathrm{O}$ dimers, form a chain of dimers along the [001] direction (Figure S5).

Detailed analysis of the intermolecular interaction energies calculated using CrystalExplorer17 [19, 20] (Figure 11) shows that the closest geometrical contacts are the most attractive in the crystal lattice of (IV) and
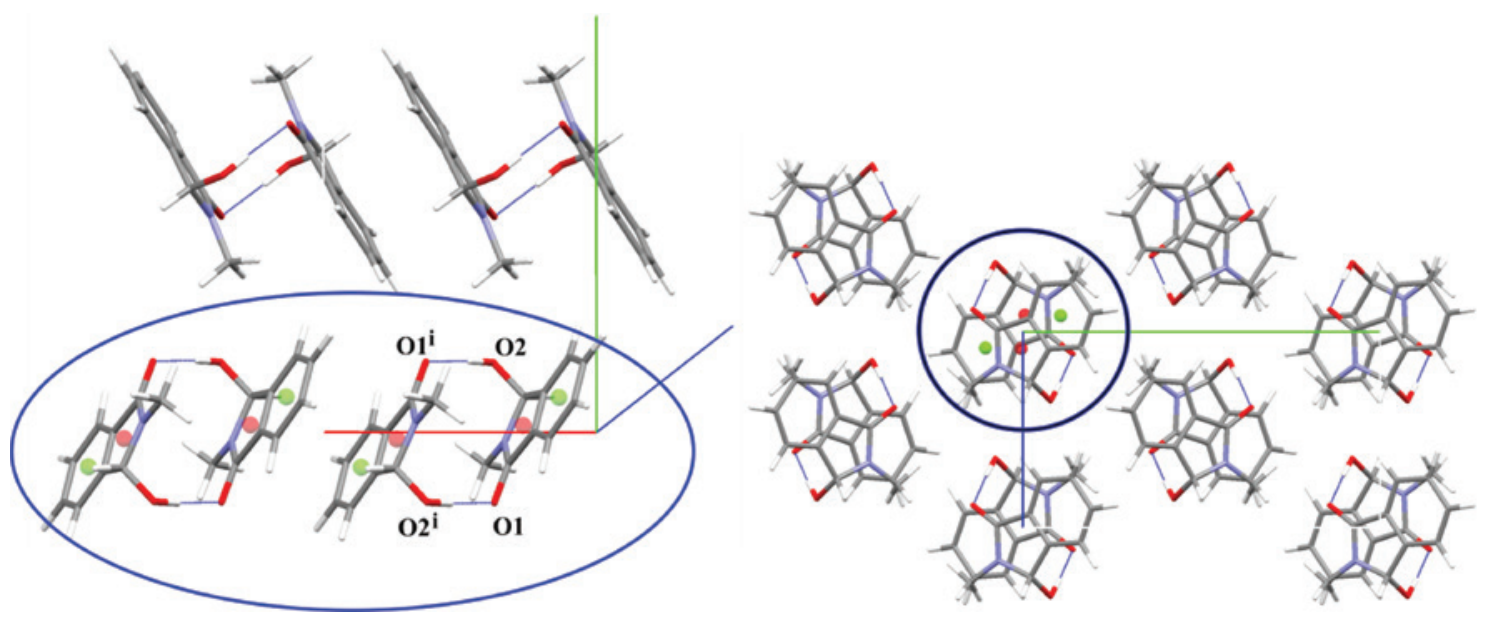

Fig. 4: Partial molecular packing of (IV) showing hydrogen-bonded dimers generated by $02-\mathrm{H} 2 \cdots 01$ interaction supported by aromatic $\pi \cdots \pi$ interactions (left); a view of the stacked ring systems along the $a$-axis (right). Red balls - centroids of 5 -membered rings of isoindole moieties ( $C g 1)$, green balls - centroids of 6 -membered rings of isoindole moieties $(C g 2)$.
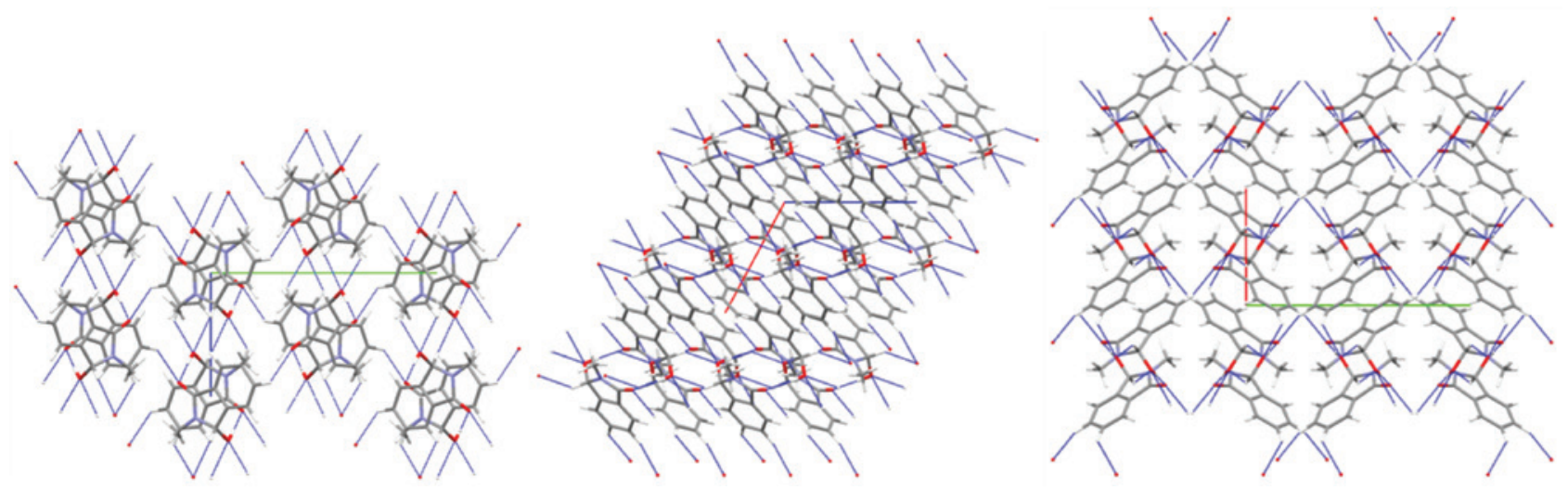

Fig. 5: Crystal packing of (IV), views along the $a, b$ and $c$-axes, respectively. 
are those with high total interaction energies. For the $\mathrm{O} 2-\mathrm{H} 2 \cdots 01$ hydrogen bond the electrostatic component is significant, and almost twice as high as the dispersion component. In contrast, in hydrogen-bonded dimers where $\pi \cdots \pi$ interactions play a key role, the dispersion component is greater than the electrostatic component.

Apart from the dimers stacked along the $a$-axis, a noticeable electrostatic contribution is propagated by the $\mathrm{C} 8-\mathrm{H} 8 \cdots \mathrm{O} 2$ interaction along the $c$-axis. The pattern of the dispersion energy is different from that of the electrostatic energy. Dispersion energy frameworks show low but meaningful values reflected by crosslinkers (contributing to total energy) between distant molecules (about $8 \AA$ A not directly connected with each other.

The representative diagram of energy frameworks for (IV), viewed down the $b$-axis, are shown in Figure 6; all remaining views are presented in Figure S26.
Compound (V): The intermolecular hydrogen bond $\mathrm{O} 2-\mathrm{H} 2 \cdots \mathrm{O} 1(1 / 2-x, 1 / 2+y, z)$ produces an infinite $z i g$ zag chain (Figure 7), similarly to structures (I) and (III) described previously. These 1D-hydrogen-bonded chains run parallel to the $b$-direction. The analysis of intermolecular packing reveals that these $\mathrm{O}-\mathrm{H} \cdots \mathrm{O}$ chain motifs are supported by aromatic $\pi \cdots \pi$ (Figure 7 ) and C9-H9B $\cdots O$ O $(1 / 2-x,-1 / 2+y, z)$ interactions (Figure S8). Figure 8 presents the complete crystal packing of (V) in the unit cell.

Other $\mathrm{C}-\mathrm{H} \cdots \mathrm{O}$ interactions (Table $\mathrm{S} 4$ and Figures S6-S7, S9-S10 in the Supplementary Material) lead to chain motifs of isoindole molecules (V), thus linking the main $\mathrm{O}-\mathrm{H} \cdots \mathrm{O}$ hydrogen-bonded chains into a network. Two interactions, $\mathrm{C} 3-\mathrm{H} 3 \cdots \mathrm{O} 2(1 / 2+x, y, 1 / 2-z)$ and C6H6 $\cdots O 1(-1 / 2+x, y, 1 / 2-z)$, bind the same molecules into a chain along [100], whereas two other contacts,

viewed along $b$ axis

(IV)
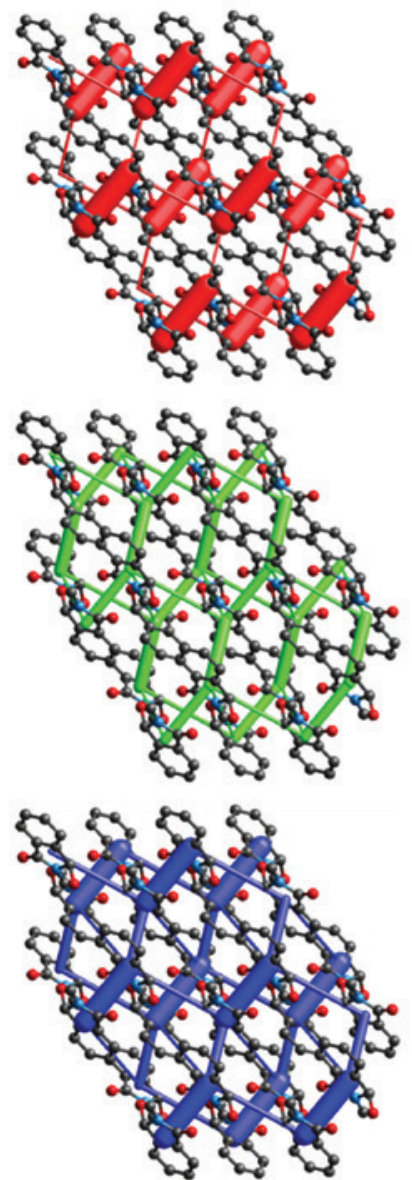

(V)
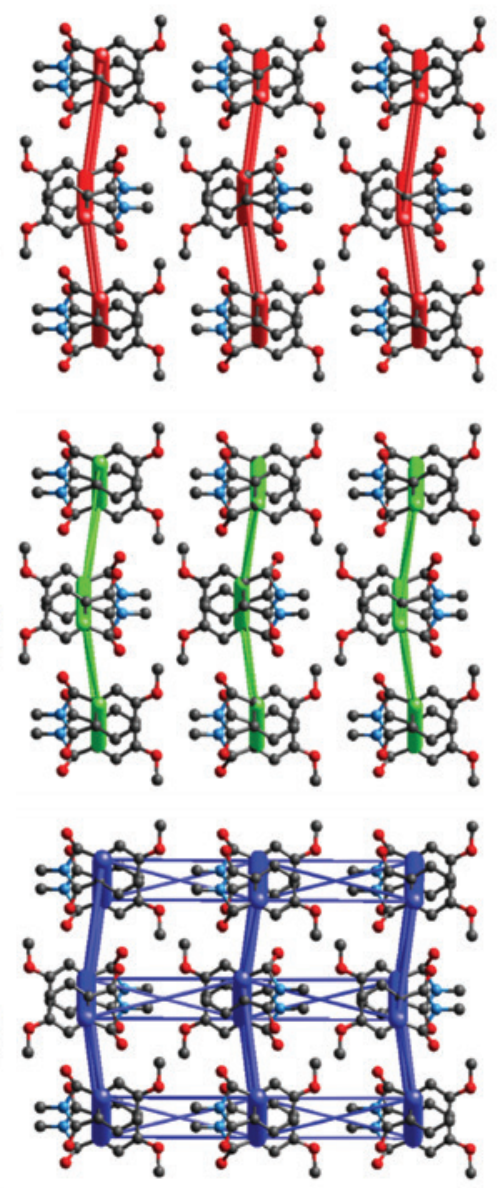

(VI)
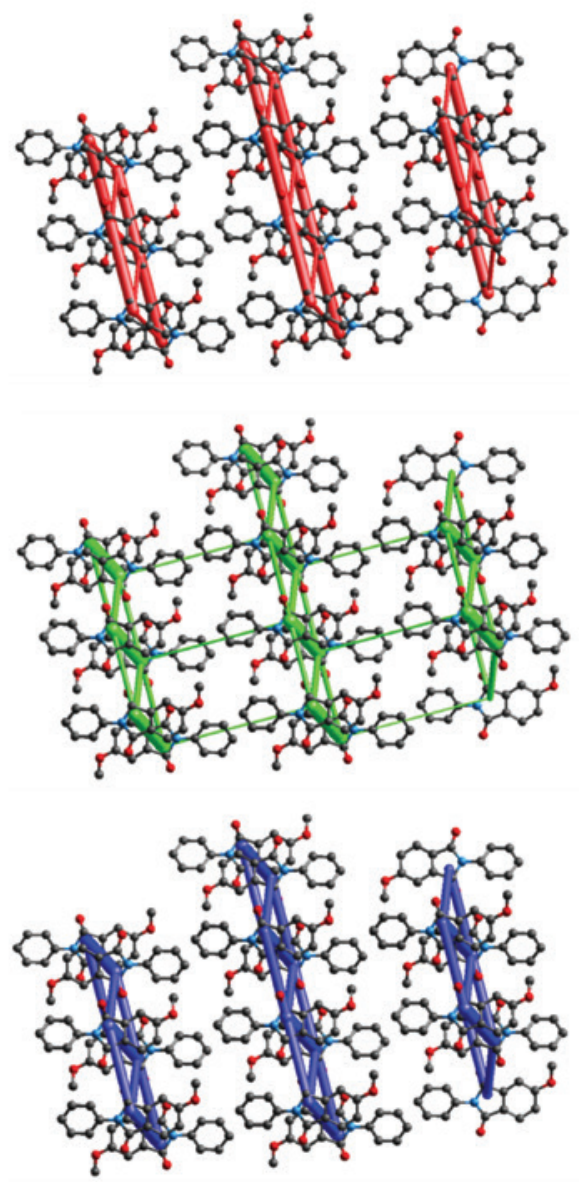

Fig. 6: The representative energy framework diagrams for separate electrostatic (red) and dispersion (green) components, and the total interaction energy (blue) selected for (IV)-(VI). All diagrams use the same energy tube scale factor of 80 and the energy threshold of $10 \mathrm{~kJ}$ mol-1. 


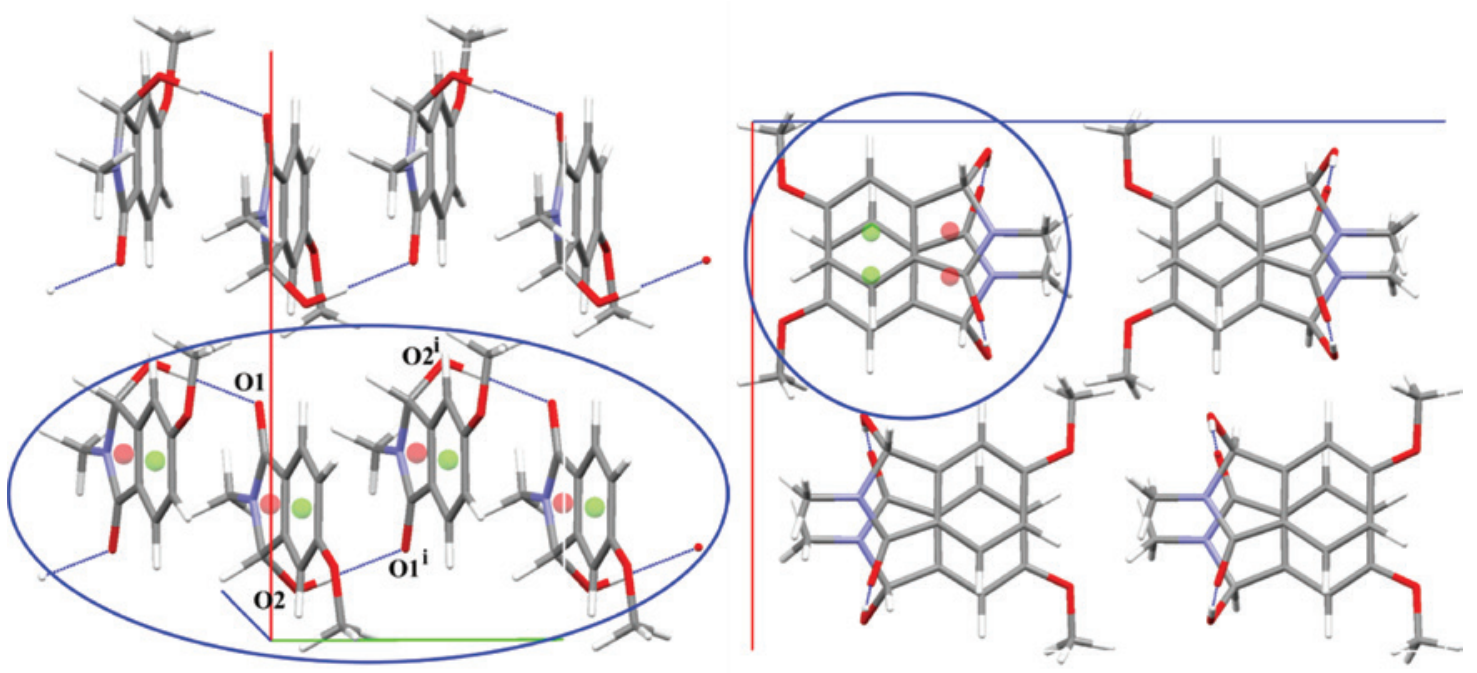

Fig. 7: Hydrogen-bonded zig-zag chains of (V) supported by aromatic $\pi \cdots \pi$ interactions (left); a view of the stacked ring systems along the $b$-axis (right). Red balls - centroids of 5-membered rings of isoindole moieties ( $C g 1)$, green balls - centroids of 6-membered rings of isoindole moieties (Cg2). Symmetry code: (i) $1 / 2-x, 1 / 2+y, z$.
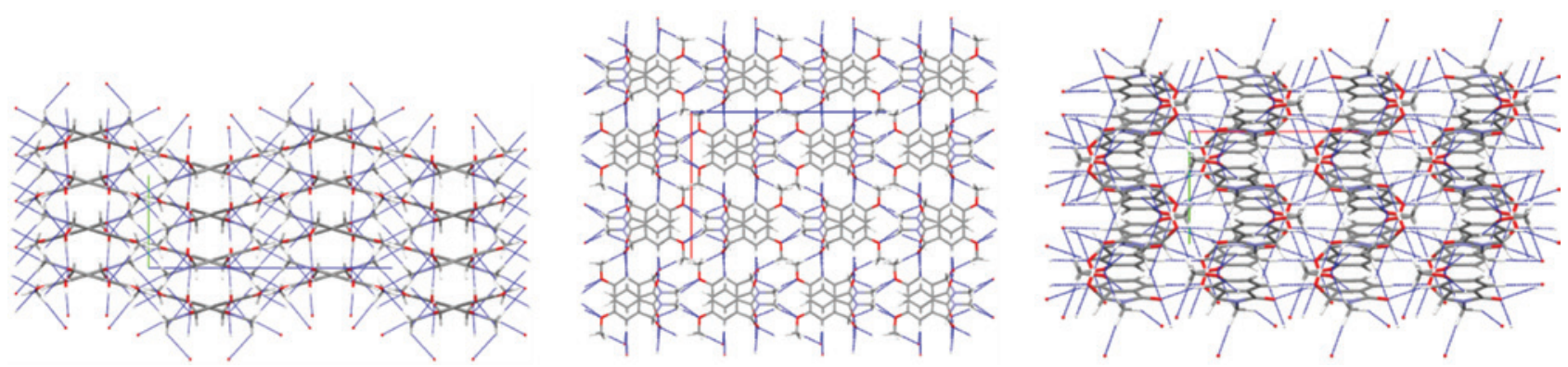

Fig. 8: Crystal packing of (V), view along the $a, b$ and $c$ axes, respectively.

C9-H9A $\cdots$ O $3(1 / 2-x,-y, 1 / 2+z)$ and C9-H9C $\cdots .03(x, 1 / 2$ $-y, 1 / 2+z)$, form independent chains of molecules along the $c$-axis.

As the $\mathrm{H} \cdots$ O distance in interaction $\mathrm{C} 33-\mathrm{H} 33 \mathrm{~A} \cdots \mathrm{O} 2$ $(-x, 1 / 2+y, 1 / 2-z)$ is rather long, it also seems to be one of the weakest, as with C9-H9B $\cdots$ O $1(1 / 2-x,-1 / 2+y, z)$. Both mentioned bridges can be seen in the [010] direction; however, the two types join different molecules, the latter of which is conjugated with an $\mathrm{O}-\mathrm{H} \cdots \mathrm{O}$ interaction.

Regarding the geometrical parameters (Table S4), more interactions are observed in the crystal lattice of compound (V) than in (IV) or (VI). However, energy indicates that only the interactions forming two main chain motifs are relevant in the crystal lattice of $(\mathbf{V})$; i.e. the $\mathrm{O} 2-\mathrm{H} 2 \cdots \mathrm{O} 1$ interaction is enhanced by $\mathrm{C} 9-\mathrm{H} 9 \mathrm{~B} \cdots \mathrm{O} 2$ and $\pi \cdots \pi$ interactions (Figure 11: INT19). The total energy of $(\mathbf{V})$ is similar to that found between molecules in the dimers of (IV) (Figure 11: INT14); however their electrostatic and dispersion contributions differ. In chains of
(V) both terms are attractive of comparable magnitude as illustrated by the corresponding thick tubes along the $b$-axis (Figure 6), whereas in the dimers of (IV) the electrostatic term is found to be clearly dominant (Figure 6). It is difficult to directly compare these intermolecular energies, as more than one interaction is responsible for them. The second important chain motif in (V) along the $c$ axis, formed by the cooperation of two contacts, H9A …03 and H9C …O3 (Figure 11: INT21 and INT22), possesses much lower interaction energy than $\mathrm{O}-\mathrm{H} \cdots \mathrm{O}$. The other three $\mathrm{C}-\mathrm{H} \cdot \mathrm{OO}$ interactions, listed in Table S4 and Figure 11, are at the edge of the energy threshold of $10 \mathrm{~kJ} \mathrm{~mol}^{-1}$ employed. The remaining intermolecular interaction energies estimated between molecules of $(\mathbf{V})$ are below $2 \mathrm{~kJ} \mathrm{~mol}^{-1}$.

Compound (VI): Similarly to derivatives (II) and (IV), compound (VI) crystallized in monoclinic $P 2_{1} / c$ space group with one molecule in the asymmetric unit (Table 1). The orientation of the hydrogen atom (H2) in the hydroxyl 
group is not the only similar feature between structures (VI) and (II) (Figure 2): despite the different proton acceptor [N2-atom in structure (II) compared to O1-atom in (VI)], both main interactions form chain motifs along the $c$-axis, with $\pi \cdots \pi$ interactions between them (Figure 9). As observed in other isoindole structures, the crystal packing of (VI) is also facilitated by $\mathrm{C}-\mathrm{H} \cdots \mathrm{O}$ hydrogen bonds (Table S6). Two intermolecular interactions, $\mathrm{C} 3-\mathrm{H} 3 \cdots \mathrm{O} 2(x, 1 / 2-y,-1 / 2+z)$ and $\mathrm{C} 96-\mathrm{H} 96 \cdots \mathrm{O} 1(x$, $1 / 2-y, 1 / 2+z)$, can be seen between molecules engaged in the $\mathrm{O}-\mathrm{H} \cdots \mathrm{O}$ chain motif (Figure S11), while another two interactions, $\mathrm{C} 8-\mathrm{H} 8 \cdots \mathrm{O} 2(1-x, 1 / 2+y, 1 / 2-z)$ and $\mathrm{C} 94-$ H94 ‥ O3 $(-1+x, y, z)$, help to construct a 3D hydrogenbonded network by expanding the chains along the $b$ and $a$ directions, respectively (Figures S12 and S13). Figure 10 presents the complete crystal packing in the unit cell.

The energy distribution patterns for compound (VI) are different from those of other presented frameworks of isoindole derivatives. Chains propagating along the $c$-axis are mainly stabilized by $\mathrm{O}-\mathrm{H} \cdots \mathrm{O}$ and $\mathrm{C}-\mathrm{H} \cdots \mathrm{O}$ interactions; however, the energy frameworks seem to be more similar to compound (IV) than to (II) (Figure 11). The dispersion energy term is approximately half of the electrostatic component, and repulsion has the most significant contribution to the overall interaction energy. One of the $\pi \cdots \pi$ inter-chain stacking interactions is characterized by a total energy value higher than those observed between dimers in structure (IV). From an energetic point of view, this $\pi \cdots \pi$ interaction is the strongest in the crystal lattice of (VI) with the dominant contribution of the dispersion term; however the electrostatic component is also significant (Figure 6: view along the $b$ direction; Figure S28; Figure 11: INT24). Interestingly, the vast majority of pairwise interactions in (VI) are of remarkably high strength $\left(40 \mathrm{~kJ} \mathrm{~mol}^{-1}\right)$.

Intermolecular model energies: Figure 11 presents the total interaction energy values, partitioned into electrostatic, dispersion and repulsion components; the polarization term has been omitted for clarity because its values are much smaller than the other contributions. The data for the six isoindole derivatives evaluated in the present study has been divided into three subgroups. Firstly, the left side block in Figure 11 summarizes the energies of

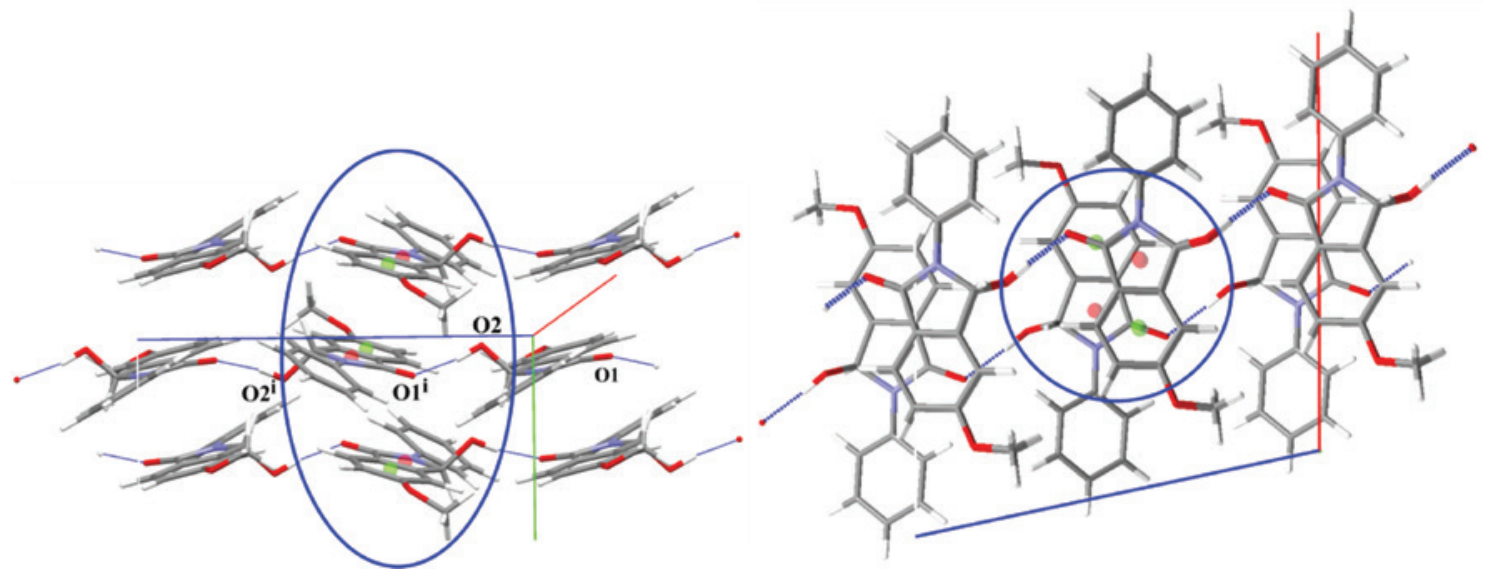

Fig. 9: Hydrogen-bonded $\mathrm{O}-\mathrm{H} \cdots \mathrm{O}$ chains in the crystallographic $c$ direction (left). Aromatic $\pi \cdots \pi$ interactions between isoindole moieties are observed between adjacent $\mathrm{O}-\mathrm{H} \cdots \mathrm{O}$ chains; stacked ring systems are seen along the $b$-axis (right). Red balls - centroids of 5 -membered rings of isoindole moieties ( $C g 1)$, green balls - centroids of 6-membered rings of isoindole moieties ( $C g 2)$. Symmetry code: (i) $x, 1 / 2-y, 1 / 2+z$.
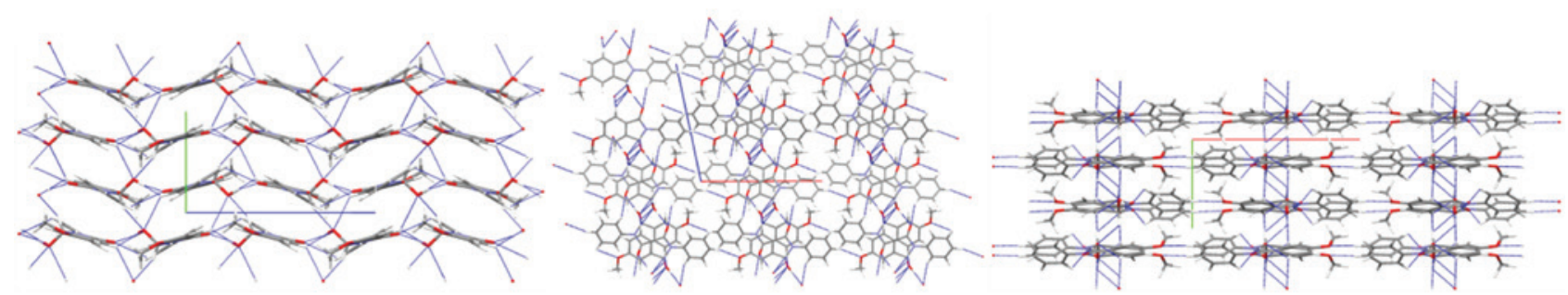

Fig. 10: Crystal packing of (VI), view along the $a, b$ and $c$-axes, respectively. 


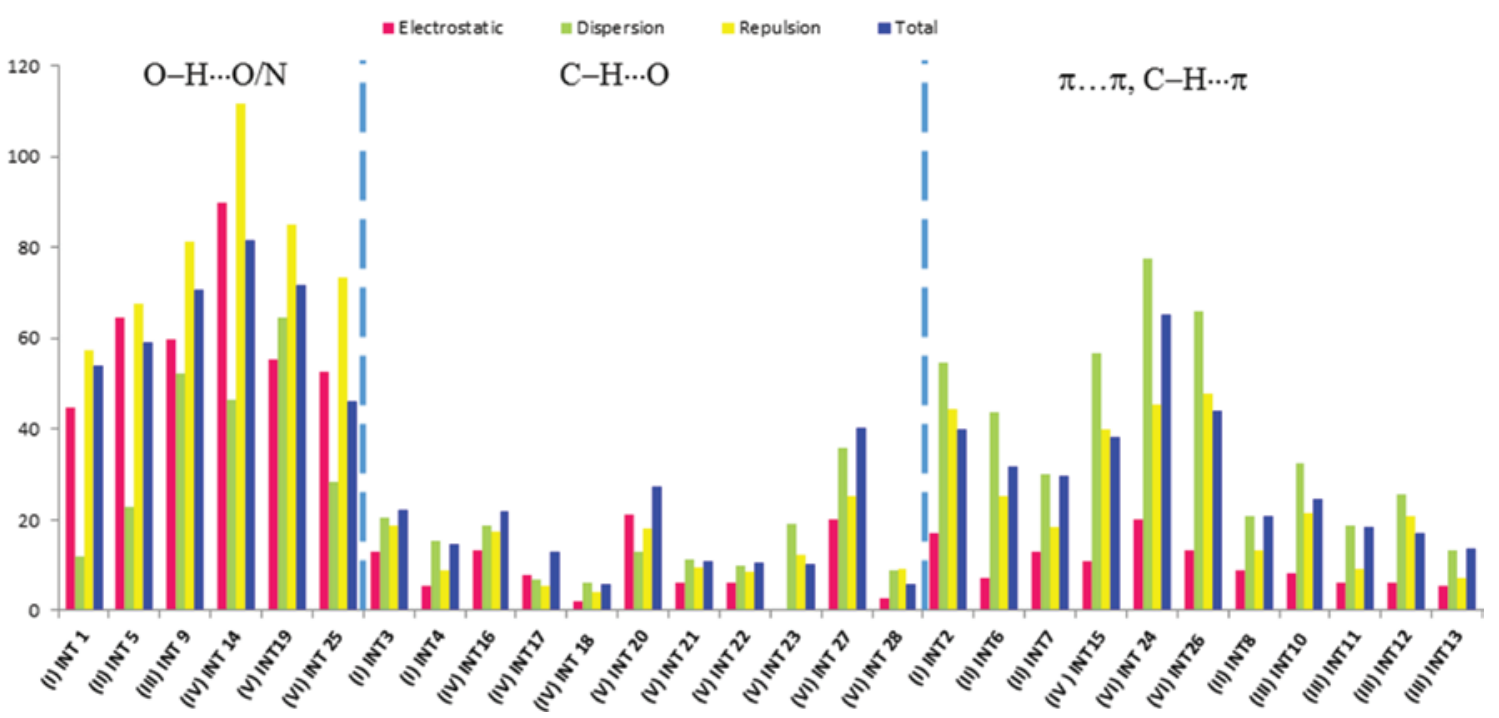

Fig. 11: Total interaction energy and its components for the pairs of molecules interacting in the crystal lattice of (I)-(VI): through $\mathrm{O}-\mathrm{H} \cdots \mathrm{O} / \mathrm{N}$ hydrogen bonds (left side block); through $\mathrm{C}-\mathrm{H} \cdots \mathrm{O}$ hydrogen bonds (middle block); with the contribution of $\pi$-electrons (right side block). All values of energies are absolute values. (Compound)-Interaction codes, left side block: (I) INT1: 02-H2 . . 01, (II) INT5: $02-\mathrm{H} 2 \cdots \mathrm{N} 2 \& \mathrm{C} 8-\mathrm{H} 8 \cdots 01 \& \mathrm{C} 96-\mathrm{H} 96 \cdots 01$, (III) INT9: 02-H2 $\cdots 01 \& \pi \cdots \pi$, (IV) INT14: 02-H2 $\cdots 01 \& \pi \cdots \pi$, (V) INT19:

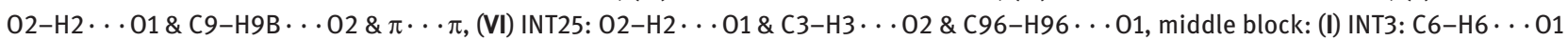

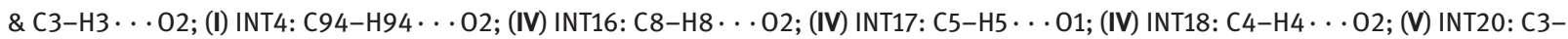

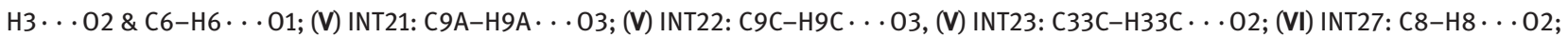
(VI) INT28: C94-H94 $\cdots$ 03, right side block: (I) INT2: $\pi \cdots \pi$ \& C8-H8 $\cdots \pi$; (II) INT6: $\pi \pi$; (II) INT7: $\pi \cdots \pi$; (IV) INT15: $\pi \cdots \pi$; (VI) INT24: $\pi \cdots \pi$; (VI) INT26: $\pi \cdots \pi$; (II) INT8: C93-H93 $\cdots \pi$; (III) INT10: C95-H95 $\cdots \pi$; (III) INT11: C93-H93 $\cdots \pi$; (III) INT12: C84-H84 $\cdots \pi$; (III) INT13: $\mathrm{C} 3-\mathrm{H} 3 \cdots \pi$.

the main $\mathrm{O}-\mathrm{H} \cdots \mathrm{O} / \mathrm{N}$ interactions found in the investigated structures; these are undoubtedly the strongest of all the hydrogen bonds, with total energies in the range of 40-80 $\mathrm{kJ} \mathrm{mol}^{-1}$. Energy partitioning clearly indicates that all of them are dominated by attractive electrostatic forces on one side and repulsive forces on the other. Dispersion contributions appear in the cases supported by the aromatic $\pi \cdots \pi$ interactions (INT9, INT14 and INT19). The greatest interaction energy obtained in the analyzed series was $80 \mathrm{~kJ} \mathrm{~mol}^{-1}$, which was estimated for the $\mathrm{O}-\mathrm{H} \cdots \mathrm{O}$ interaction of the hydrogen-bonded dimer in (IV). For the sake of comparison, strong H-bonds in carboxylic acid dimers have interaction energies around $60-70 \mathrm{~kJ} \mathrm{~mol}^{-1}$ [47].

The middle block in Figure 11 shows a comparison of the characteristics of the $\mathrm{C}-\mathrm{H} \cdots \mathrm{O}$ pairwise intermolecular energies. The energy value of the strongest $\mathrm{C}-\mathrm{H} \cdots \mathrm{O}$ interaction (INT27) is comparable to that of the weakest $\mathrm{O}-\mathrm{H} \cdots \mathrm{O}$ hydrogen bond (INT1), which suggests that $\mathrm{C} 8-\mathrm{H} 8 \cdots \mathrm{O} 2$ in (VI) has a similar importance in building the hydrogen-bonded network as $\mathrm{O} 2-\mathrm{H} 2 \cdots \mathrm{O} 1$ in (I). The strengths of other $\mathrm{C}-\mathrm{H} \cdots \mathrm{O}$ pairwise interactions (INT3, INT16, INT20) are about half that of the strongest interaction of this type (INT27), or definitely smaller (from 6 to $15 \mathrm{~kJ} \mathrm{~mol}^{-1}$ ); this is in good agreement with the values given in previous studies. Although no clear trend is visible describing the electrostatic and dispersive terms of the investigated isoindole structures, it becomes apparent that the latter is often greater than the former.

The right side block in Figure 11 presents the third type of intermolecular interactions in isoindole structures, namely interactions with the contribution of $\pi$-electrons. As predicted by Hirshfeld surface analysis, they play an important role in the investigated crystal packings of (I)-(VI). Surprisingly, one of the stacked molecular pairs was found to exhibit an intermolecular energy as high as $65 \mathrm{~kJ} \mathrm{~mol}^{-1}$ (INT24), whereas the energies of the remaining interactions with $\pi$-electron contributions range from 14 to $44 \mathrm{~kJ} \mathrm{~mol}^{-1}$. For the purposes of comparison, displacedstacking interactions in naphthalene are estimated to be $16 \mathrm{~kJ} \mathrm{~mol}^{-1}$, while the intra-chain stacking interactions in 3,4-dichlorophenol are $17 \mathrm{~kJ} \mathrm{~mol}^{-1}$ and those in 4-bromo3-chlorophenol are $19 \mathrm{~kJ} \mathrm{~mol}^{-1}$ [18]. The characteristic feature of $\pi$-interactions is their attractive dispersive nature. Their repulsion contributions are smaller and electrostatic forces usually below the threshold level of $10 \mathrm{~kJ} \mathrm{~mol}^{-1}$. 
Tab. 2: Some statistics based on pairwise model energies for (I)-(VI).

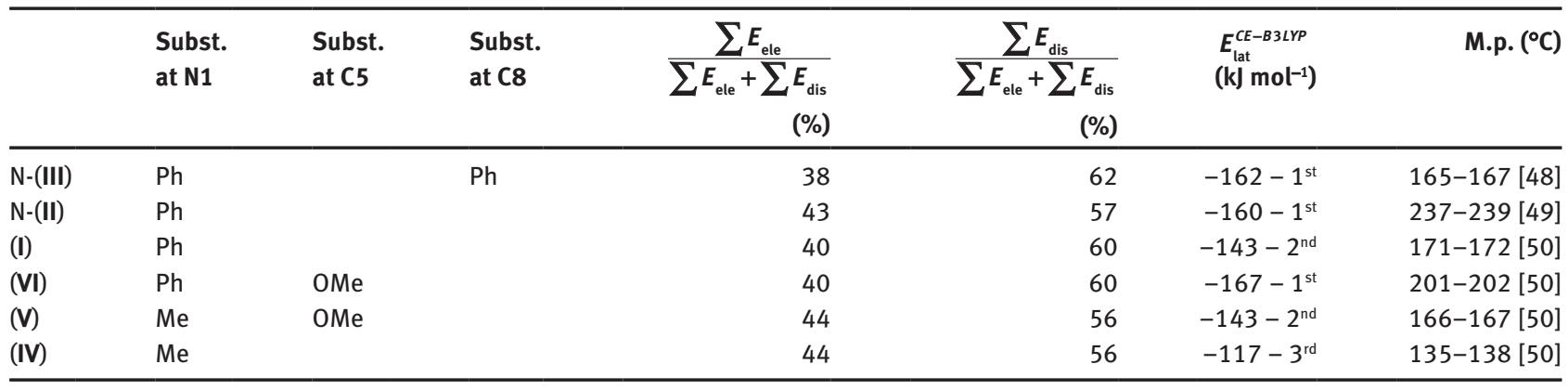

A deeper insight into the hierarchy of interactions can be obtained by analyzing the separate electrostatic and dispersion contributions to the interaction energy. Since, the model energies incorporate scaled components, appropriate scale factors $(k)$ were used in calculations of $\sum E_{\text {ele }}$ and $\sum E_{\text {dis }}, 1.057$ and 0.871 , respectively [20]. Thus, the percentage values shown in Table 2 represent the proportions of electrostatic and dispersion energies that contribute to the sums over the molecular pairs in the cluster of $8 \AA$. The breakdown confirms the dispersion component predominates in the crystal structures of (I)-(VI). Considering all pairwise interactions, it can be seen that the ratio of electrostatic to dispersion energy is $c a$. 2:3. Indeed, some molecular pairs were found with no obvious directional intermolecular interaction between them, but with a significant dispersive energy component; for example, three different pairs of molecules in structure (I) are characterized by dispersion energy ranging from 6 to $12 \mathrm{~kJ}$ $\mathrm{mol}^{-1}$, while the electrostatic component is negligible.

According to the latest approach presented by Thomas et al. [51] the lattice energy $\left(E_{\text {lat }}^{C E-B L Y P}\right)$ can be estimated using CE-B3LYP pairwise model energies $\left(E_{\text {tot }}^{A B}\right)$, obtained from CrystalExplorer17 [19, 20], by direct summation of them:

$$
E_{\text {lat }}^{C E-B 3 L Y P}=0.5 \sum_{R_{A B}<R} E_{\text {tot }}^{A B} .
$$

The CE-B3LYP lattice energies calculated for experimental crystal structures of (I)-(VI) are summarized in Table 2. For three isoindole structures, (VI), (III) and (II), lattice energies are comparable and much more negative, than for structure (IV). The melting points of studied isoindole crystals are related to the estimated lattice energies; more negative $E_{\text {lat }}^{C E-B 3 L Y P}$ higher the melting point. Relatively lower melting point of (IV) compared to (II) and (VI) reflects the lower lattice energy associated with smaller conformational changes within the $N$-methyl group rather than the $N$-phenyl one. In case of structure (III), for which the relationship of the lattice energy to the melting point seems to be disturbed, some additional structural features should be considered. Compound (III) crystallizes, as the only one from six studied isoindole derivatives, in a noncentrosymmetric space group $P 2_{1} 2_{1} 2_{1}$, and the absolute configuration of the asymmetric $\mathrm{C} 8$ atom was determined as $(S)$ [14]. As we discussed earlier, the molecular structure of (III) differs from the other $N$-phenyl derivatives described here i.e. the dihedral angle of 55.98(1) ${ }^{\circ}$ indicates the significant bending of the $N$-phenyl ring with respect to isoindole moiety and the longest N1-C8 bond length. The bulky substituent at $\mathrm{C} 8$ atom has also a crucial impact on the gas-phase molecular structure of (III) that the chain (open, acyl-amide) form of isoindole moiety is energetically preferred compared to ring (close, hydroxylactam) form (as observed in investigated crystals); the difference in energy values between ring and chain tautomers is $28 \mathrm{~kJ} \mathrm{~mol}^{-1}$ based on geometry optimizations at B3LYP/6-311++G(3df,3pd) level of theory. For comparison, the corresponding difference in energy between ring and chain tautomers of $-33 \mathrm{~kJ} \mathrm{~mol}^{-1}$ for derivative (IV) indicates that hydroxy-lactam tautomer predominates.

\section{Conclusions}

Energy framework are useful tools and shift the focus of investigation from chemical bonds to intermolecular interactions. From the analysis of the hierarchy of intermolecular interactions in three crystal structures rather general conclusion emerge that will have validity for a wider range of similar organic compounds. Most importantly the role of non-directional dispersion interactions is often significant and might exceed the importance of more directional classical hydrogen bonds.

For exercises in proposed "crystal engineering" this means that a rational design of a crystal structure from "synthons" will not be giving a unique result in many cases, a problem that increases in importance alongside 
molecular complexity. Therefore crystal engineering seems a concept with limited value.

A quantitative analysis of intermolecular interaction energies of a structure can be helpful in this respect, since a probabilistic treatment of the likelihood of a particular directional interaction could help future efforts toward successful crystal engineering and provide a yield in analogy to an organic synthesis.

Acknowledgements: The medium-resolution X-ray measurements were carried out within the project I-20090042 EC at the light source DORIS III at HASYLAB/DESY, Hamburg, Germany. LC would like to thank the Deutscher Akademischer Austauschdienst (DAAD) for a 2-month fellowship. Professor Simon Grabowsky is gratefully acknowledged for stimulating discussions and his help with the program CrystalExplorer17. We would also like to thank Professor Mark A. Spackman for his constructive suggestions. The authors declare no competing financial interests.

\section{References}

[1] G. R. Desiraju, Crystal Gazing: structure prediction and polymorphism. Science. 1997, 278, 404.

[2] A. Nangia, G. R. Desiraju, Supramolecular synthons and pattern recognition. Top. Curr. Chem. Design Organic Solids. 1998, $198,57$.

[3] G. R. Desiraju, Supramolecular synthons in crystal engineering - a new organic synthesis. Angew. Chem. Int. Ed. Engl. 1995, 34, 2311.

[4] T. Eicher, S. Hauptmann, The Chemistry of Heterocycles; Structure, Reactions, Syntheses, and Applications. Wiley-VCH, Weinheim, 2003.

[5] T. L. Gilchrist, Heterocyclic Chemistry. Prentice Hall Press, NJ, 1997.

[6] J. A. Joule, K. Mills, Heterocyclic chemistry. Blackwell Publishing, Oxford, UK, 2010.

[7] M. Anzini, A. Cappelli, S. Vomero, G. Giorgi, T. Langer, G. Bruni, M. R. Romeo, A. S. Basile, Molecular basis of peripheral vs central benzodiazepine receptor selectivity in a new class of peripheral benzodiazepine receptor ligands related to alpidem. J. Med. Chem. 1996, 39, 4275.

[8] T. Wada, N. Fukuda, Pharmacologic profile of a new anxiolytic, DN-2327: effect of Ro15-1788 and interaction with diazepam in rodents. Psychopharmacology. 1991, 103, 314.

[9] L. A. Sorbera, P. A., Leeson, J. Silvestre, J. Castaner, Pagoclone. Drugs Future. 2001, 26, 651.

[10] V. Gotor, R. Limeres, R. Garcia, M. Bayod, R. Brieva, Enzymatic resolution of ( \pm )-6-(5-chloropyridin-2-yl)-6-vinyloxy-carbonyloxy-6,7-dihydro[5H]pyrrolo[3,4-b]pyrazin-5-one. Synthesis of (+)-zopiclone. Tetrahedron Asymmetr. 1997, 8, 995.

[11] C. Cabrele, O. Reiser, The modern face of synthetic heterocyclic chemistry. J. Org. Chem. 2016, 81, 10109.
[12] A. P. Taylor, R. P. Robinson, Y. M. Fobian, D. C. Blakemore, L. H. Jones, 0 . Fadeyi, Modern advances in heterocyclic chemistry in drug discovery. Org. Biomol. Chem. 2016, 14, 6611.

[13] W. R. Pitt, D. M. Parry, B. G. Perry, C. R. Groom, Heteroaromatic rings of the future. J. Med. Chem. 2009, 52, 2952.

[14] L. Chęcińska, S. Grabowsky, M. Małecka, A. J. Rybarczyk-Pirek, A. Jóźwiak, C. Paulmann, P. Luger, Experimental and theoretical electron-density study of three isoindole derivatives: topological and Hirshfeld surface analysis of weak intermolecular interactions. Acta Crystallogr. B. 2011, 67, 569.

[15] R. F. W. Bader, Atoms in molecules. A quantum theory. Clarendon Press, Oxford, 1990.

[16] M. A. Spackman, D. Jayatilaka, Hirshfeld surface analysis. CrystEngComm. 2009, 11, 19.

[17] M. J. Turner, S. Grabowsky, D. Jayatilaka, M. A. Spackman, Accurate and efficient model energies for exploring intermolecular interactions in molecular crystals. J. Phys. Chem. Lett. 2014, 5, 4249.

[18] M. J. Turner, S. P. Thomas, M. W. Shi, D. Jayatilaka, M. A. Spackman, Energy frameworks: insights into interaction anisotropy and the mechanical properties of molecular crystals. Chem. Commun. 2015, 51, 3735.

[19] M. J. Turner, J. J. McKinnon, S. K. Wolff, D. J. Grimwood, P. R. Spackman, D. Jayatilaka, M. A. Spackman, CrystalExplorer17. University of Western Australia, 2017.

[20] C. F. Mackenzie, P. R. Spackman, D. Jayatilaka, M. A. Spackman, CrystalExplorer model energies and energy frameworks: extension to metal coordination compounds, organic salts, solvates and open-shell systems. IUCr) 2017, 4, 575.

[21] A. Gavezzotti, Calculation of lattice energies of organic crystals: the PIXEL integration method in comparison with more traditional methods. Z. Kristallogr. 2005, 220, 499.

[22] B. Dittrich, T. Koritsanszky, P. Luger, A simple approach to nonspherical electron densities by using invarioms. Angew. Chem. Int. Ed. 2004, 43, 2718.

[23] B. Dittrich, C. B. Hübschle, K. Pröpper, F. Dietrich, T. Stolper, J. J. Holstein, The generalized invariom database (GID). Acta Crystallogr. B. 2013, 69, 91.

[24] F. H. Allen, I. J. Bruno, Bond lengths in organic and metalorganic compounds revisited: $\mathrm{X}-\mathrm{H}$ bond lengths from neutron diffraction data. Acta Crystallogr. B. 2010, 66, 380.

[25] W. Kabsch, Automatic processing of rotation diffraction data from crystals of initially unknown symmetry and cell constants. J. Appl. Crystallogr. 1993, 26, 795.

[26] W. Kabsch, XDS. Acta Crystallogr. D. 2010, 66, 125.

[27] G. M. Sheldrick, SADABS, University of Göttingen, Germany, 2008.

[28] Bruker, XPREP. Bruker AXS Inc., Madison, Wisconsin, USA, 2005.

[29] G. M. Sheldrick, SHELXT - integrated space-group and crystal-structure determination. Acta Crystallogr. A. 2015, $71,3$.

[30] G. M. Sheldrick, Crystal structure refinement with SHELXL. Acta Crystallogr. C. 2015, 71, 3.

[31] L. J. Farrugia, WinGX and ORTEP for Windows: an update. J. Appl. Crystallogr. 2012, 45, 849.

[32] C. B. Hübschle, P. Luger, B. Dittrich, Automation of invariom and of experimental charge density modelling of organic molecules with the preprocessor program. Invariom Tool. J. Appl. Crystallogr. 2007, 40, 623. 
[33] A. Volkov, P. Macchi, L. J. Farrugia, C. Gatti, P. Mallinson, T. Richter, T. Koritsanszky, XD2006 (Ver. 5.42 from $18^{\text {th }}$ September 2007). University of New York at Buffalo, NY, USA, 2006.

[34] J. Lübben, C. Volkmann, S. Grabowsky, A. Edwards, W. Morgenroth, F. P. A. Fabbiani, G. M. Sheldrick, B. Dittrich, On the temperature dependence of $\mathrm{H}-U_{\text {iso }}$ in the riding hydrogen model. Acta Crystallogr. A. 2014, 70, 309.

[35] A. L. Spek, Structure validation in chemical crystallography. Acta Crystallogr. D. 2009, 65, 148.

[36] C.F. Macrae, I. J. Bruno, J. A. Chisholm, P. R. Edgington, P. McCabe, E. Pidcock, L. Rodriguez-Monge, R. Taylor, J. van de Streek, P. A. Wood, Mercury CSD 2.0 - new features for the visualization and investigation of crystal structures. J. Appl. Crystallogr. 2008, 41, 466.

[37] M. J. Frisch, G. W. Trucks, H. B. Schlegel, G. E. Scuseria, M. A. Robb, J. R. Cheeseman, G. Scalmani, V. Barone, B. Mennucci, G. A. Petersson, H. Nakatsuji, M. Caricato, X. Li, H. P. Hratchian, A. F. Izmaylov, J. Bloino, G. Zheng, J. L. Sonnenberg, M. Hada, M. Ehara, K. Toyota, R. Fukuda, J. Hasegawa, M. Ishida, T. Nakajima, Y. Honda, O. Kitao, H. Nakai, T. Vreven, J. A. Montgomery Jr, J. E. Peralta, F. Ogliaro, M. J. Bearpark, J. Heyd, E. N. Brothers, K. N. Kudin, V. N. Staroverov, R. Kobayashi, J. Normand, K. Raghavachari, A. P. Rendell, J. C. Burant, S. S. lyengar, J. Tomasi, M. Cossi, N. Rega, N. J. Millam, M. Klene, J. E. Knox, J. B. Cross, V. Bakken, C. Adamo, J. Jaramillo, R. Gomperts, R. E. Stratmann, O. Yazyev, A. J. Austin, R. Cammi, C. Pomelli, J. W. Ochterski, R. L. Martin, K. Morokuma, V. G. Zakrzewski, G. A. Voth, P. Salvador, J. J. Dannenberg, S. Dapprich, A. D. Daniels, Ö. Farkas, J. B. Foresman, J. V. Ortiz, J. Cioslowski, D. J. Fox, Gaussian09, Revision D.01, Gaussian, Inc.: Wallingford, CT, USA, 2013.

[38] T.A. Keith, AIMAll (Ver. 16.05.18) TK Gristmill Software, Overland Park KS, USA, 2014, (aim.tkgristmill.com).

[39] L. Chęcińska, S. Mebs, C. B. Hübschle, D. Förster, W. Morgenroth, P. Luger, Reproducibility and transferability of topological data: experimental charge density study of two modifications of L-alanyl-L-tyrosyl-L-alanine. Org. Biomol. Chem. 2006, 4, 3242.

[40] M. A. Spackman, J. J. McKinnon, Fingerprinting intermolecular interactions in molecular crystals. CrystEngComm. 2002, 4, 378.

[41] J. J. McKinnon, D. Jayatilaka, M. A. Spackman, Towards quantitative analysis of intermolecular interactions with Hirshfeld surfaces. Chem. Commun. 2007, 37, 3814.
[42] J. J. McKinnon, M. A. Spackman, A. S. Mitchell, Novel tools for visualizing and exploring intermolecular interactions in molecular crystals. Acta Crystallogr. B. 2004, 60, 627.

[43] M. A. Spackman, Molecules in crystals. Phys. Scr. 2013, 87, 048103.

[44] M. W. Shi, S. G. Stewart, A. N. Sobolev, B. Dittrich, T. Schirmeister, P. Luger, M. Hesse, Y.-S. Chen, P. R. Spackman, M. A. Spackman, S. Grabowsky, Approaching an experimental electron-density model of the biologically active trans-epoxysuccinyl amide group - substituent effects vs. crystal packing. J. Phys. Org. Chem. 2017, 30, e3683.

[45] E. Eikland, M. A. Spackman, B. B. Iversen, Quantifying hostguest interaction energies in clathrates of Dianin's compound. Cryst. Growth Des. 2016, 16, 6858.

[46] S. Bhandary, K. S. R. N. Mangalampalli, U. Ramamurty, D. Chopra, Crystal structure-mechanical property correlations in $\mathrm{N}$-(3-ethynylphenyl)-3-fluorobenzamide polymorphs. Cryst. Growth Des. 2018, 18, 47.

[47] J. D. Dunitz, A. Gavezzotti, Supramolecular synthons: validation and ranking of intermolecular interaction energies. Cryst. Growth Des. 2012, 12, 5873.

[48] J. Epsztajn, A. Józwiak, K. Czech, A. K. Szczesniak, Application of organolithium and related reagents in synthesis, part VI. A general study of the lithiation of secondary picoline- and isonicotine amides. Monatsh. Chem. 1990, 121, 909.

[49] J. Epsztajn, A. Józwiak, A. K. Szczesniak, Application of organolithium and related reagents in synthesis. XV. A concise regiospecific conversion of picolinic- and isonicotinic acids into 2-benzoyl- and 4-benzoylnicotinic acids. Synth. Commun. 1994, 24, 1789.

[50] A. Jóźwiak, M. Ciechańska, The behavior of 2-substituted3-hydroxyisoindolinones in the reaction with sec-butyllithium. J. Heterocycl. Chem. 2013, 51, 357.

[51] S. P. Thomas, P. R. Spackman, D. Jayatilaka, M. A. Spackman, Accurate Lattice Energies for Molecular Crystals from Experimental Crystal Structures. J. Chem. Theory Comput. 2018, 14, 1614.

Supplementary Material: The online version of this article offers supplementary material (https://doi.org/10.1515/zkri-2018-2076). 